\title{
Expert Database of Unified Parameter Adjustment Based on Long-step Calibration and Cubic Spline Interpolation
}

\author{
Liao Tianfa ${ }^{*}$, Xue Jiaxiang \\ School of Mechanical and Automotive Engineering, South China University of Technology, 510640 Guangzhou,China
}

\begin{abstract}
There are many kinds of aluminum alloys suitable for double-pulse MIG welding, however, the welding parameters are much more and they cannot be easily adjusted. Thus, it is necessary to establish the expert database for the welding source. In order to improve the adjustment performance to precisely control the welding current, this paper proposes a kind of expert database of unified parameter adjustment based on the long-step calibration and cubic spline interpolation to make the welding parameters "continuously adjusted" and "precisely matched" as well as make the test results verified.
\end{abstract}

\section{Introduction}

The double-pulse MIG welding involves a large amount of control parameters, moreover, it would directly affect the stability and joint performance in the welding process whether the parameters selected are suitable or not. Therefore, it is an important content for the welding study to establish an expert database including welding conditions, methods and parameters. However, there is much less study on the expert database of double-pulse MIG welding of aluminium alloy at home. This paper establishes an expert database to make the welding parameters "continuously adjusted" and "precisely matched" by a specific algorithm based on the welding process test [1,2], which has a very important significance to study the high-end double-pulse MIG welding source of aluminium alloy.

\section{Unified Database}

It is the key for double-pulse MIG welding of aluminium alloy that the wire feed rate matches well with the current during the welding process. However, the difficulties to establish the double-pulse MIG welding expert database of aluminium alloy are as follows. (1) There are many kinds of aluminium alloys, as well as different wire feed rates are needed according to the different welding process requirements. So the database establishment would cost much more manpower, time and money. (2) The aluminium alloy has better conductive and heat-conducting properties. When the aluminium alloys are welded with the same welding parameters at different temperatures, the welding results are significantly different. This results in worse repeatability of welding data and worse consistency [3]. The original data are needed to establish the expert database, and they present as some discrete points in a two-dimensional plane. Then these discrete points are processed into a line by the interpolation algorithm or curve fitting approximation, thus obtaining the complete continuous curve. Finally, another parameter is found out to match with some parameter to establish a unified database $[4,5]$.
There are two methods to build the continuous model, namely, interpolation method and curve fitting. The interpolation method requires that the interpolation function curve must go through these discrete points, but the curve fitting only requires that the fitting function curve approaches the known discrete points as far as possible as well as the fitting function curve can accord with the distribution profile of known discrete points [5].

It is assumed that the continuous function is in the form of $f \in[a, b]$. The simple function $\varphi \in \Phi$ in the finite-dimensional linear subspace $\Phi$ (or polynomial space $\mathrm{P}_{\mathrm{n}}$ ) is close to the continuous function $\mathrm{f}$, and two kinds of measurement standards for the approximating function $\varphi$ are proposed as follows.

(1) Best square approximation

$$
\text { For } f \in[a, b], \varphi^{*} \in \Phi=\operatorname{Span}\left\{\varphi_{0}, \varphi_{1}, \ldots, \varphi_{n}\right\}
$$

should be worked out to make

$$
\int_{a}^{b}\left[f(x)-\varphi^{*}(x)\right]^{2} d x=\inf _{\varphi \in \phi} \int_{a}^{b}[f(x)-\varphi(x)]^{2} d x
$$

Where inf is the infimum sign.

(2) Best uniform approximation

$$
\text { For } f \in C[a, b], p \in p_{n}=\operatorname{Span}\left\{1, x, x^{2}, \ldots, x^{n}\right\}
$$

should be worked out to make

$$
\max _{a \leq x \leq b}\left|f(x)-p^{*}(x)\right|=\inf _{p \in p_{n}}\left[\max _{a \leq x \leq b}|f(x)-p(x)|\right]
$$

Then the best uniform approximation is also called as Chebyshev approximation.

\section{Long-step calibration method}

At present, the intelligent welding source with the parameter storage only has a certain automatic control functions, and they store much more welding parameters by a large amount of welding procedure tests. However, in the practical welding process, the existing welding parameters can only conduct the read operations rather than the write operations. This would result in two problems, as follows. (1) The huge number of data are needed, and the welding parameters which are not stored cannot be used for welding. But if all the welding data are stored, the cost is greatly high; (2) There possibility exist the "global risk", for example, if there exists local

\footnotetext{
liaotianfa@163.com
} 
difference in a type of welding sources, all the welding parameters are invalid so that the welding fails. In order to solve these problems, the new generation of doublepulse MIG welding source of aluminium alloy not only can store the welding parameters but also has the selflearning function.

So this paper proposes a kind of parameter selflearning method based on the long-step calibration, and then the expert database which only has read operation has the dynamically flexible self-adjusting function. This lays a foundation for studying the new generation of double-pulse MIG welding source of aluminium alloy.

The long-step calibration refers to the larger step among the data, and it only calibrates some typical parameters. For example, the scope of welding current for the double-pulse MIG welding of aluminum welding wire with a diameter of $1.6 \mathrm{~mm}$ is [40A], so the step is set as $40 \mathrm{~A}$, then the welding parameter to be calibrated is 40A. If there exists some special parameters such as inflection point, and so on, the refinement calibration is needed near the special points.

\section{Cubic spline interpolation algorithm}

Equations should be centred and should be numbered with the number on the right-hand side.

In order to overcome the Runge's phenomenon of Lagrange interpolation curve as well as improve the smoothness of Newton interpolation in the nodes, this paper uses the cubic spline interpolation algorithm.

The principle of cubic spline interpolation algorithm is as follows. There are interpolation nodes in the interval [a,b], and they meet $a=x_{0}<x_{1}<\ldots<x_{n}<x_{n+1}=b . f(x)$ is the function in the interval [a,b], and if $S(x)$ meets the following three conditions:

(1) it goes through $(\mathrm{n}+1)$ interpolation points, namely, $S\left(x_{i}\right)=f\left(x_{i}\right)=y_{i}(i=0,1,2, \ldots, n)$;

(2) it is second-order continuous, namely, $S(x) \in C^{2}[a, b]$;

(3) It is cubic subsection, namely, namely, the function $S(x)$ is all the cubic polynomial in each interval $\left[a, x_{1}\right],\left[x_{1}, x_{2}\right], \ldots,\left[x_{n}, x_{n+1}\right] . f(x)$ as well as its first and second derivatives are continuous for $x_{i}(i=1,2, \ldots, n)$, then $S(x)$ is called as cubic spline interpolation function of $f(x)$.

Let $M_{i}=S^{\prime \prime}\left(x_{i}\right)$, then after twice integrals for the interpolation condition $S\left(x_{i}\right)=y_{i},(i=0,1,2, \ldots, n)$ and $S^{\prime \prime}(x)=\frac{x-x_{i}}{-h_{i-1}} M_{i-1}+\frac{x-x_{i-1}}{h_{i-1}} M_{i}(i=1,2, \ldots, n)$, the expression of cubic spline interpolation function $S\left(x_{i}\right)=y_{i},(i=0,1,2, \ldots, n)$ could be obtained.

$$
\begin{gathered}
S(x)=\frac{M_{i-1}}{6 h_{i-1}}\left(x_{i}-x\right)^{3}+\frac{M_{i}}{6 h_{i-1}}\left(x-x_{i-1}\right)^{3}+\left[\frac{y_{i-1}}{h_{i-1}}-\frac{M_{i-1}}{6} h_{i-1}\right]\left(x_{i}-x\right)+\left[\frac{y_{i-1}}{h_{i-1}}-\frac{M_{i-1}}{6} h_{i-1}\right]\left(x-x_{i-1}\right) \\
, \quad x_{i-1} \leq x \leq x_{i},(i=1,2, \ldots n)
\end{gathered}
$$

Where $h_{i-1}=x_{i}-x_{i-1}$.

Because the function $S(x)$ has the second derivative in the sampling point $x_{i}$, the natural boundary conditions $S^{\prime \prime}\left(x_{0}\right)=y_{0}^{\prime}=0$ and $S^{\prime}\left(x_{n}\right)=y_{n}{ }_{n}=0$ are increased according to the cubic natural spline interpolation.

The equation set is obtained as follows.

$$
\left[\begin{array}{ccccc}
2 & u_{0} & & & \\
\lambda_{1} & 2 & u_{1} & & \\
& \ddots & \ddots & \ddots & \\
& & \lambda_{n-1} & 2 & u_{n-1} \\
& & & \lambda_{n} & 2
\end{array}\right] \circ\left[\begin{array}{c}
M_{0} \\
M_{1} \\
\cdots \\
M_{n-1} \\
M_{n}
\end{array}\right]=\left[\begin{array}{c}
U_{0} \\
U_{1} \\
\cdots \\
U_{n-1} \\
U_{n}
\end{array}\right]
$$

$$
\left\{\begin{array}{c}
u_{i}=\frac{h_{i+1}}{h_{i}+h_{i+1}} \\
\lambda_{i}=1-u \\
\text { Where } \\
\left.U_{i=1,2, \ldots, n-1 ;} ; u_{o=0} ; U_{0}=0 ; y_{n+1}-y_{i}-\frac{y_{1}+y_{i-1}}{h_{i+1}}\right] ; \lambda_{n}=0 。
\end{array}\right.
$$

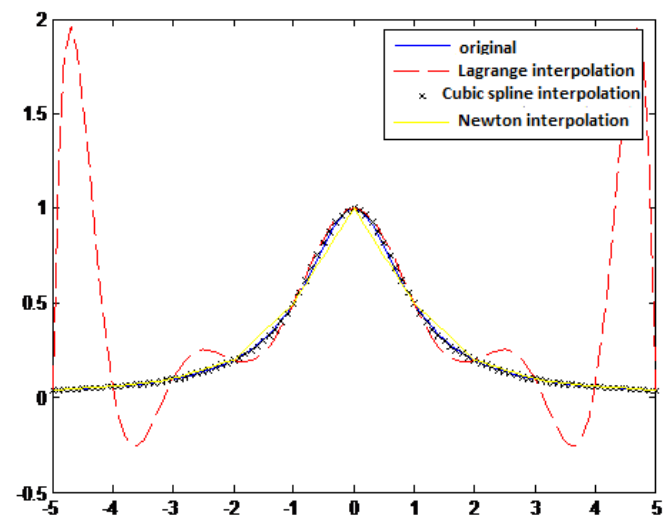

(a) The graph of cubic spline interpolation curve

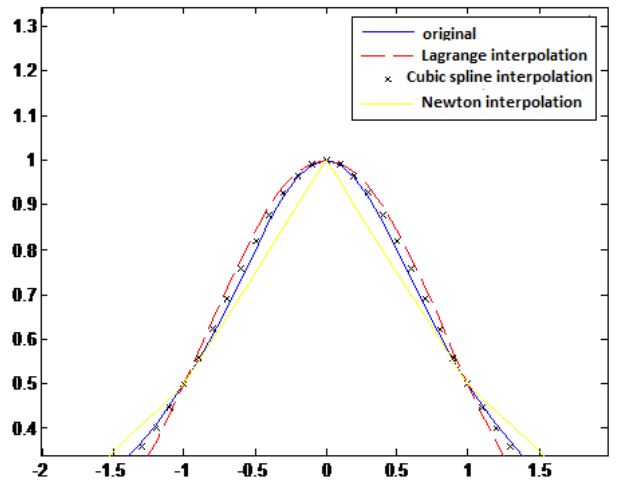

(b) The partial enlargement graph of Node

Fig.1 Graph of cubic spline interpolation algorithm

Figure 2 is the flow chart of cubic spline interpolation algorithm. 


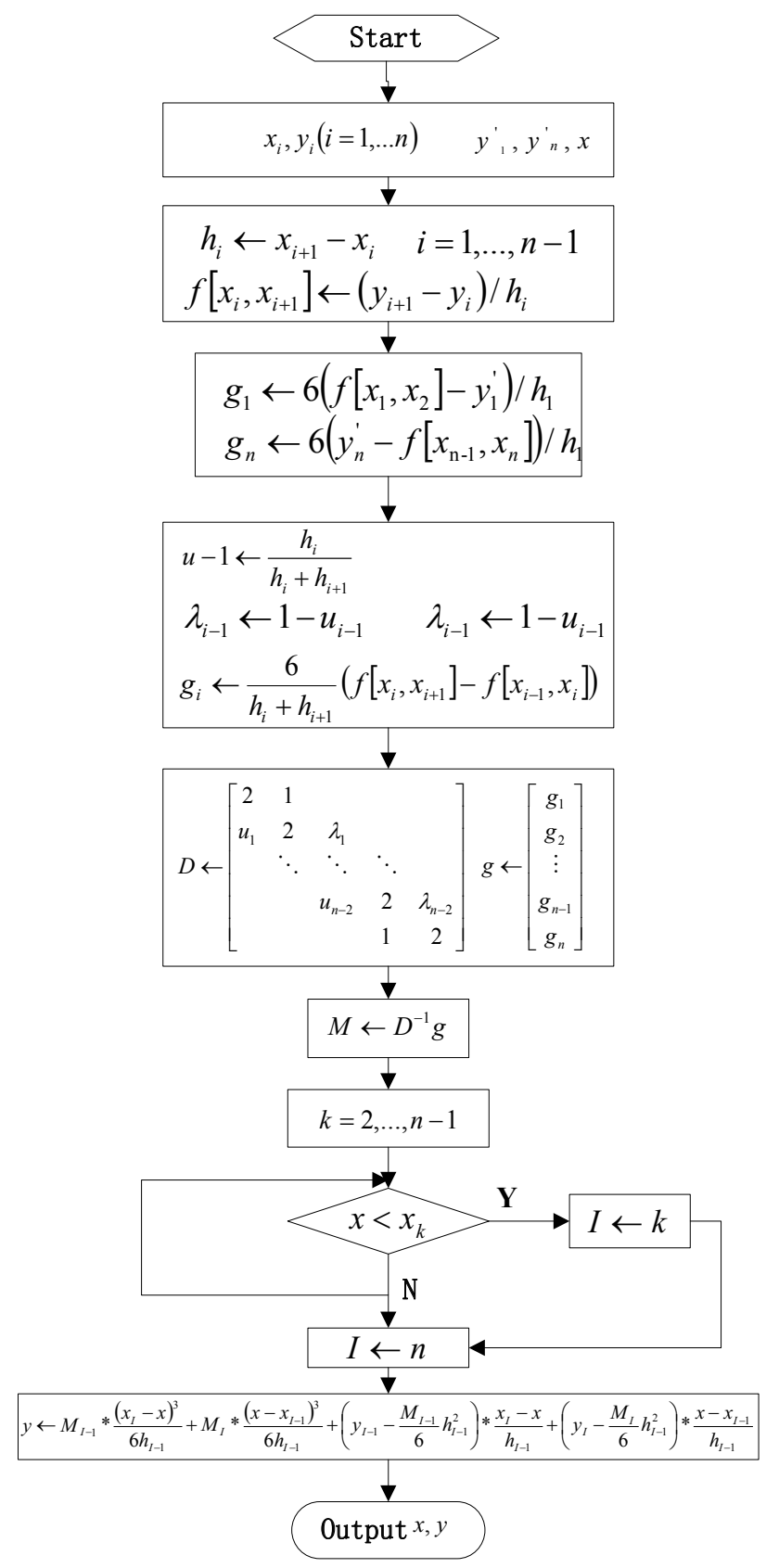

Fig.2 Flow chart of cubic spline interpolation algorithm

The equation set is solved to figure out $M_{i}(i=0,1,2, \ldots, n)$, then the solved $M_{i}(i=0,1,2, \ldots, n)$ is substituted into $S(x)$. Thus the cubic natural spline interpolation function in each subarea $\left[x_{i-1}, x_{i}\right](i=1,2, \ldots, n)$ can be obtained.

During the process of actual programming, if the values of $x_{i}, y_{i}(i=1, \ldots, n)$ and $y^{\prime}, y_{n}^{\prime}, x$ are known, when the cubic spline interpolation algorithm is used to figure out $\mathrm{x}$ and $\mathrm{y}$ values, the solution is shown as the flow diagram in Figure 1.

The Matlab software is used to obtain the cubic natural spline interpolation curve of function $f(x)=\frac{1}{1+x^{2}}, x \in[-5,5]$, as shown in figure 1. From
Figure 1, the cubic natural spline interpolation algorithm well overcomes the Runge's phenomenon of Lagrange interpolation algorithm, roughness in the nodes of Newton interpolation algorithm, and so on, the interpolation curve drawn is close to the original curve, as well as the interpolation curve is very smooth.

\section{Result}

After the text edit has been completed, the paper is ready for the template. Duplicate the template file by using the Save As command, and use the naming convention prescribed by your conference for the name of your paper. In this newly created file, highlight all of the contents and import your prepared text file. You are now ready to style your paper.

Figure 3 is the expert database established by the cubic natural spline interpolation algorithm, and the parameters selected from the expert database are used for the welding line of aluminium alloy by the double-pulse MIG welding. Moreover, the original instantaneous current and voltage waveforms are collected by the dynamic wavelet analyzer in the welding process, and then the U-I diagram is analyzed.

Figure $3 a$ and $b$ are the original instantaneous current and voltage waveforms in the double-pulse MIG welding of aluminium alloy, respectively. Clearly, the pulse waveforms dispaly the sine-wave modulation, and the instantaneous current and voltage waveforms can directly reflect the stability of welding process.

Figure $3 \mathrm{c}$ shows the U-I diagram after the wavelet filter. The edge line group keeps in order, their distribution is very concentrated, and the U-I diagram presents the high repeatability. Thus the welding quality and welding process control are stable and reliable.

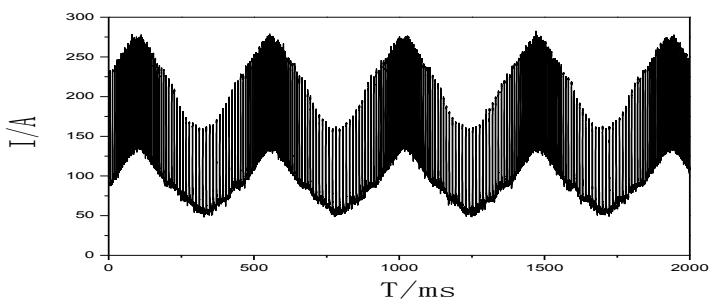

(a) The instantaneous waveform of original welding current

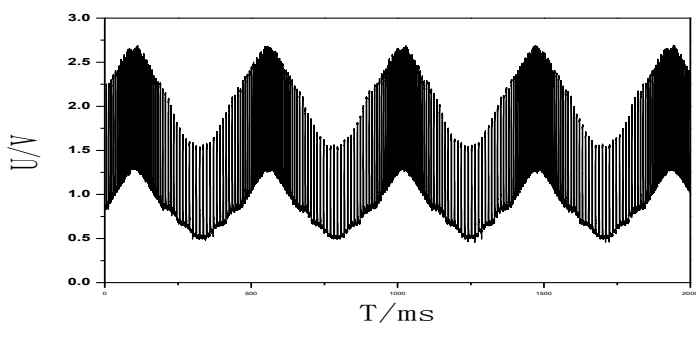

(b) The instantaneous waveform of original welding voltage 


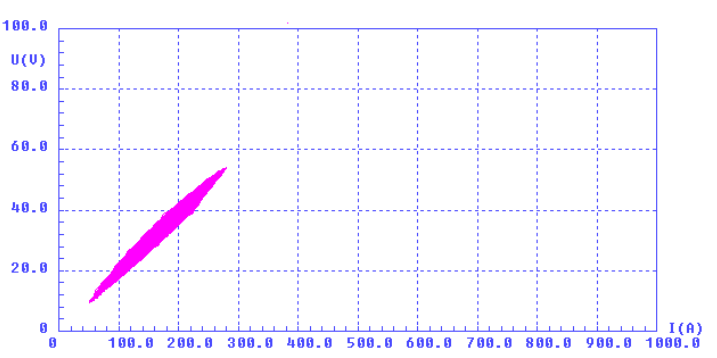

(c) The instantaneous U-I figure of welding

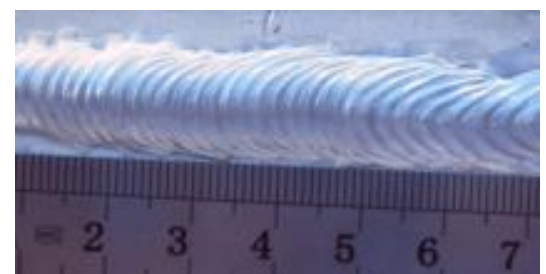

(d) The photo of weld

Fig.3 Welding test results based on cubic spline interpolation algorithm

Figure $3 \mathrm{~d}$ shows the welding lines photos, in which the welding lines with clear fish-scale pattern corresponding to the sine waveform are displayed.

It is suggested by the test process and the results that the welding parameters obtained by the cubic spline interpolation algorithm are stable and reliable in the double-pulse MIG welding of aluminium alloy, and the welding lines are in fish-scale patterns. So these parameters can meet the welding requirement of aluminium alloy.

\section{Conclusion}

The cubic spline interpolation algorithm can well overcome the disadvantages such as Runge's phenomenon, roughness in the nodes, and so on. In this paper, the expert database of unified parameter adjustment based on the long-step calibration and cubic spline interpolation is proposed. The long-step calibration can make the welding parameters "continuously adjusted", the cubic spline interpolation can make the welding parameters"precisely matched", and thus the welding source has the unified adjustment function. This greatly decreases the repetitive process adjustment steps and meets the actual demand in the welding process.

\section{References}

1. Yao Ping, Xue Jiaxiang, Meng Wanjun, etc. The process parameters of aluminium alloy double pulse weld forming $[\mathrm{J}]$.The influence of welding journal, 2009, 30(3):69-72.

2. Xi Shengfeng. The research and application of data fitting program [J].Journal of Zhejiang University of Technology, 2003, 31(5):587-590.

3. Zheng Xianyi, Yao Yangxin, Lei Xiuren, etc. The application of numerical analysis [M].Guangzhou: South China University of Technology Press, 2008.
4. Chen Wenliu, Wang Ziyang. Fitting of cubic spline interpolation in engineering application $[\mathrm{J}]$. Journal of China normal university: natural science edition, 2004, 38(4):418-422.

5. Yang Y U, Yuan J H, Qian J, et al. Cubic Spline Interpolation with New Boundary Conditions[J]. Computer Engineering \& Software, 2016. 